\title{
Expression and clinical significance of FOXE1 in papillary thyroid carcinoma
}

\author{
YOUBEN FAN $^{1,2}$, ZHENG DING $^{1,2}$, ZHILI YANG ${ }^{1,2}$, XIANZHAO DENG $^{1,2}$, \\ $\mathrm{JIE} \mathrm{KANG}^{1,2}$, BO WU ${ }^{1,2}$ and QI ZHENG ${ }^{1,2}$ \\ ${ }^{1}$ Department of Surgery, Affiliated Sixth People's Hospital; ${ }^{2}$ Medical Center of Thyroid Disease, \\ Shanghai Jiaotong University, Shanghai 200233, P.R. China
}

Received January 14, 2013; Accepted April 22, 2013

DOI: $10.3892 / \mathrm{mmr} .2013 .1494$

\begin{abstract}
Papillary thyroid cancer (PTC) is the most common form of well-differentiated thyroid carcinoma. Although the incidence of PTC has been on the increase in China, the precise causes of the disease remain unknown and a prognostic index has yet to be identified. In the present study, quantitative real-time PCR and western blot analysis were used to detect the mRNA and protein expression levels, respectively, of forkhead box E1 (FOXE1) in 30 PTC tissue specimens matched with adjacent non-tumor thyroid tissues. FOXE1 expression in 81 PTC paraffin-embedded blocks was also evaluated retrospectively by immunohistochemistry (IHC) and its correlation with the clinicopathological parameters of PTC patients was analyzed. Compared with adjacent non-tumor tissues, the mRNA expression levels of the FOXE1 gene in tumor tissues were significantly higher in $65 \%$ of PTC tissue specimens. The results from western blotting and IHC analysis were consistent with FOXE1 mRNA expression. Furthermore, the expression of FOXE1 was significantly correlated with the extra-capsular invasion of tumor cells, lymph node metastasis and tumor stage $(\mathrm{P}=0.048, \mathrm{P}=0.036$ and $\mathrm{P}=0.009$, respectively). Thus, our results demonstrated that the expression of FOXE1 was significantly increased in PTC tumor tissues and correlated with clinical prognosis. Therefore, FOXE1 is a potential biomarker for PTC prognosis as well as a new therapeutic target.
\end{abstract}

\section{Introduction}

Papillary thyroid cancer (PTC) accounts for $80-85 \%$ of thyroid cancer cases (1). The clinical prognosis for the majority of cases is satisfactory; however, $14 \%$ of cases demonstrate relatively early recurrence and others present with severe inva-

Correspondence to: Dr Youben Fan, Department of Surgery, Affiliated Sixth People's Hospital, Shanghai Jiaotong University, Lane 600, Yishan Road, Shanghai 200233, P.R. China

E-mail: fanyouben2006@163.com

Key words: papillary thyroid carcinoma, FOXE1 expression, clinical significance sion, multiple lymph node metastasis and occasionally, distant metastasis (2). Currently, extremely few biological markers that are useful for the diagnostic and prognostic analysis of PTC have been identified.

Forkhead box E1 [FOXE1, formerly known as thyroid transcription factor 2 (TTF2)] belongs to a large family of transcription factors characterized by a distinct forkhead domain and is an important thyroid-specific factor. Numerous members of the forkhead family are potent transcriptional activators in the adult thyroid and are important in cell growth and differentiation $(3,4)$, including FOXE1 which is important in the development and differentiation of thyroid follicular cells $(5,6)$. In addition, a growing body of data have indicated that FOXE1 is important in the initiation of specific tumors, including pancreatic cancer, cutaneous squamous cell carcinoma and thyroid neoplasms (7-9). Furthermore, previous studies demonstrated that FOXE1 transcripts are detectable in PTC (10), and a recent genome-wide association study showed that a common variant on $9 \mathrm{q} 22.33$ led to an increased risk of papillary and follicular thyroid carcinoma (11). Notably, the FOXE1 gene is located on human chromosome 9q22.

To the best of our knowledge, the expression of the FOXE1 gene and its correlation with the clinicopathological parameters of PTC patients has not been previously reported. Thus, in the present study, we investigated FOXE1 expression, and then analyzed the correlation between FOXE1 expression and the clinicopathological parameters in PTC.

\section{Materials and methods}

Tumor specimens and patient information. PTC and adjacent non-tumor thyroid tissue specimens were collected immediately following surgical removal and stored at $-80^{\circ} \mathrm{C}$ until use. A total of 30 pairs of fresh-frozen PTC tissues and adjacent non-tumor tissues were used for the analysis of FOXE1 gene expression by quantitative real-time PCR (qPCR) and western blotting. An additional 81 paraffin-embedded tissue blocks of PTC were obtained from the Department of Pathology, Affiliated Sixth People's Hospital, Shanghai Jiaotong University (Shanghai, China) between 2010 and 2012, and were randomly selected for IHC analysis.

The average age of the patients from which the 81 PTC cases were derived was 49.6 years (range, 25-73 years) 
Table I. PCR primers and conditions.

\begin{tabular}{llcr}
\hline Gene & \multicolumn{1}{c}{ Primer sequence } & Temperature $\left({ }^{\circ} \mathrm{C}\right)$ & Product size $($ bp $)$ \\
\hline FOXE1 & F-GCTGGTTTTCCCTGTCTCTG & 60 & 100 \\
& R-AGATGGGGGAGACTGAAGGT & 60 & \\
& F-TTGTTACAGGAAGTCCCTTGCC & 60 & 101 \\
& R-ATGCTATCACCTCCCCTGTGTG & 61 & \\
\hline
\end{tabular}

FOXE1, forkhead box E1; F, forward; R, reverse.

and included 27 males and 54 females. The study protocol and consent form were approved by the Institutional Ethics Committee of the Affiliated Sixth People's Hospital, Shanghai Jiaotong University. All patients were informed of the aims of the study and provided written informed consent.

RNA isolation and qPCR assay. Total RNA was extracted from PTC and adjacent non-tumor tissue specimens using TRIzol reagent (Invitrogen Life Technologies, Carlsbad, CA, USA) according to the manufacturer's instructions. First strand cDNA was then synthesized using the Reverse Transcription Reagent kit (Takara Bio, Inc., Dalian, China). The qPCR assay was conducted in a $10-\mu 1$ reaction mixture according to the SYBR-Green PCR kit (Takara Bio, Inc.) and analyzed in a 96-well plate using the Applied Biosystems 7500 Real Time PCR system (Applied Biosystems, Foster City, CA, USA). The primer sequences used in this study are shown in Table I. The PCR conditions were as follows: $95^{\circ} \mathrm{C}$ for $15 \mathrm{sec}$, followed by 40 cycles at $95^{\circ} \mathrm{C}$ for $5 \mathrm{sec}$ and $60^{\circ} \mathrm{C}$ for $34 \mathrm{sec}$. $\beta$-actin was used as an internal control. Relative values of transcripts were calculated using the formula: $2^{-\Delta \Delta \mathrm{Ct}}$. Experiments were performed in triplicate.

Western blotting. To evaluate the level of FOXE1 protein expression, tissue lysates were prepared from fresh-frozen PTC and adjacent non-tumor tissue specimens by centrifugation at $12,000 \mathrm{x} \mathrm{g}$ for $20 \mathrm{~min}$ at $4^{\circ} \mathrm{C}$. The concentration of the protein lysate was then determined using BCA reagent (Beyotime, Shanghai, China). For each sample, a volume equivalent to $20-\mu \mathrm{g}$ protein lysate was separated by SDS-PAGE followed by transfer onto a nitrocellulose membrane (Bio-Rad, Hercules, CA, USA). The membrane was blocked with $5 \%$ non-fat milk for $1 \mathrm{~h}$ and then incubated with rabbit anti-FOXE1 monoclonal antibody (1:1000; Abcam, Cambridge, MA, USA) overnight at $4^{\circ} \mathrm{C}$. After washing in Tris-buffered saline with Tween- 20 to remove excess primary antibody, the blots were incubated for $1 \mathrm{~h}$ with a specific secondary antibody (goat anti-rabbit IgG, 1:5,000; Santa Cruz Biotechnology, Inc., CA, USA). Antibody binding was detected using the enhanced chemiluminescent reagents (Pierce Biotechnology, Inc., Rockford, IL, USA) and measured using Kodak Scientific Imaging Systems (New Haven, CT, USA). A goat-specific monoclonal GAPDH antibody (1:5,000; Bioworld Technology, Minneapolis, MN, USA) was used as a control.

IHC analysis. In total, 81 paraffin-embedded tissue blocks of PTC were retrieved for IHC analysis. The blocks were cut into
4- $\mu \mathrm{m}$ sections, deparaffinized with xylene and rehydrated in a graded ethanol series. Antigen retrieval was performed by boiling tissue sections in EDTA solution (1:50) for $20 \mathrm{~min}$. The sections were incubated with the primary antibody, rabbit antiFOXE1 monoclonal antibody (1:250; Abcam) overnight at $4^{\circ} \mathrm{C}$. The slides were then incubated with a biotinylated secondary antibody (goat anti-rabbit $\mathrm{IgG}$ ) at $37^{\circ} \mathrm{C}$ for $30 \mathrm{~min}$. The slides were then stained with hematoxylin and eosin (H\&E) for 2 min, dehydrated, mounted and imaged using the Microscope ScanScope (Olympus, Tokyo, Japan).

Immunohistological scores and clinicopathological parameters. Two pathologists blinded to the identity of the specimens, examined the percentage of positively stained cells in contrast to the total section area (TSA $=100 \%$ ). They also assessed the intensity of the immunostained slides and scored them as previously described (12). Based on the percentage of positive cells, the level of staining was defined as follows: $0 \%$, negative (-); 1-33\%, weak (+); 34-66\%, moderate (++) and 67-100\%, strong (+++). The intensity of the immunoreactions was scored as follows: 0 , negative $(-) ; 1$, weak $(+) ; 2$, moderate $(++)$ and 3 , strong $(+++)$. The total scores in the tumor and non-tumor regions were determined as the sum of the products of the positivity and intensity grades. A total score of $>2.0$ was considered to represent a strong positive score.

Clinicopathological parameters, including age, gender, tumor size, extra-capsular invasion, multifocality, lymph node metastasis, distant metastasis and tumor stage (TNM classification) were analyzed $(13,14)$. Correlations between the IHC results and the clinicopathological parameters were evaluated.

Statistical analysis. The differences between the FOXE1 expression levels of PTC and adjacent non-tumor tissue specimens were determined statistically using the Mann-Whitney $\mathrm{U}$ test or the independent samples t-test. Correlations between FOXE1 expression and the clinicopathological parameters of PTC patients were analyzed using the Pearson's Chi-squared test. Statistical analyses were performed using SPSS software (version 17.0; SPSS, Inc., Chicago, IL, USA). P<0.05 was considered to indicate a statistically significant result.

\section{Results}

FOXE1 mRNA expression in PTC. Out of the 30 pairs of specimens investigated by qPCR, 26 paired results were analyzed. The average mRNA expression levels of FOXE1 


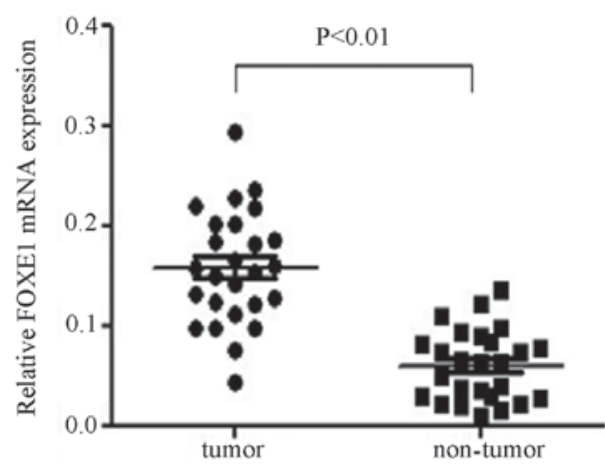

Figure 1. mRNA expression levels of FOXE1 in PTC (tumor) and adjacent non-tumor (non-tumor) tissues were examined by qRT-PCR. Data represent the mean of triplicate experiments normalized to $\beta$-actin expression. PTC, papillary thyroid cancer; FOXE1, forkhead box E1; qRT-PCR, quantitative real-time PCR.

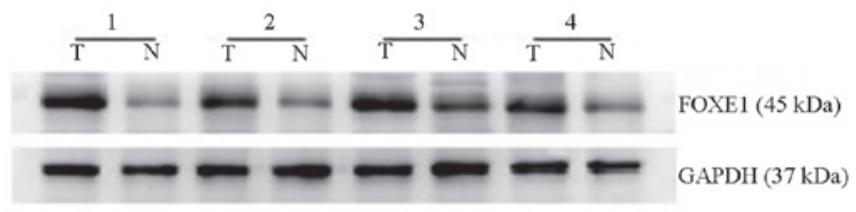

Figure 2. Protein expression levels of FOXE1 in PTC and adjacent non-tumo thyroid tissues were examined by western blotting. GAPDH protein expression levels were measured as a control. T, tumor tissue; N, non-tumor tissue; PTC, papillary thyroid cancer; FOXE1, forkhead box E1.

were significantly higher in PTC tissues compared with adjacent non-tumor thyroid tissues $(0.1575 \pm 0.0566$ versus $0.0598 \pm 0.0350, \mathrm{P}<0.01)$, equating to an $\sim 2.6$-fold increase in the expression of FOXE1 in PTC tissues (Fig. 1). The results also demonstrated that $65 \%$ (17 out of 26) of PTC tissues expressed higher levels of FOXE1 compared with the matched adjacent non-tumor thyroid tissues.

FOXE1 protein expression levels in PTC versus non-tumor thyroid tissues. FOXE1 protein expression levels were also analyzed by western blotting. FOXE1 protein levels in PTC tissues were significantly higher compared with the matched adjacent non-tumor thyroid tissues. FOXE1 was more markedly expressed in 58\% (15 out of 26) of PTC tissues compared with the paired non-tumor tissues (Fig. 2). Thus, these results are consistent with the data obtained by the qPCR assay.

FOXE1 protein staining indices by IHC. IHC staining was performed to analyze FOXE1 expression in paraffin-embedded tissue samples of PTC. The results demonstrated that FOXE1 staining was widely expressed in all tumor regions of the 81 PTC tissue sections (100\%); however, it was present in only 31 out of 81 samples in the non-tumor regions (38.3\%). A higher degree of FOXE1 staining was evident in the tumor regions compared with the corresponding non-tumor tissues (Fig. 3). Additionally, FOXE1 staining was predominantly present in cell nuclei. However, in non-tumor thyroid follicular epithelial cells, FOXE1 staining was negative or exhibited a weak level of staining. Thus, FOXE1 expression is significantly elevated at the RNA and protein levels in PTC.
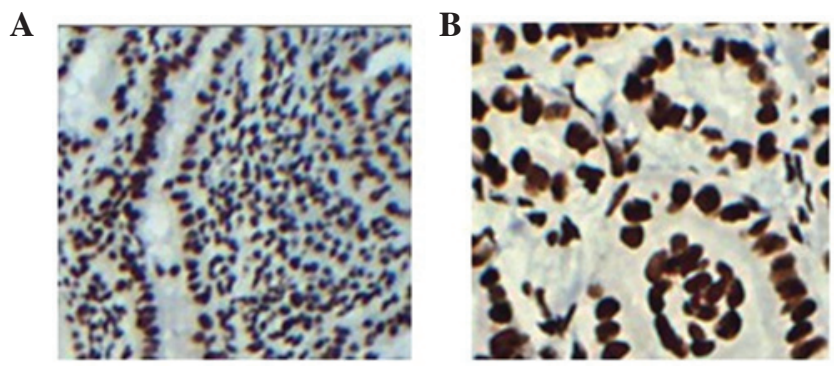

C
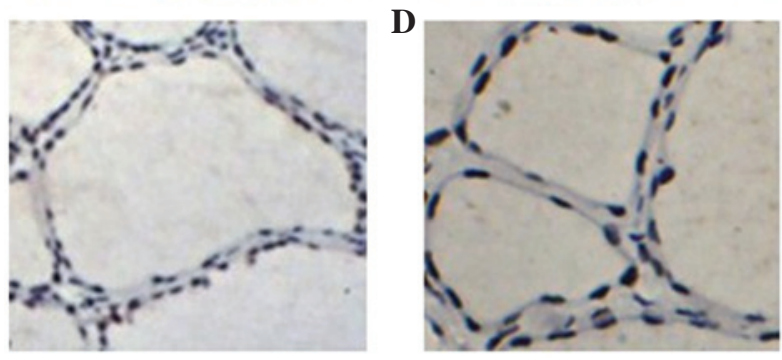

Figure 3. Immunohistochemical staining of FOXE1 in PTC tissue sections. FOXE1 expression was examined in paraffin-embedded sections of PTC by immunohistochemical staining. (A and B) PTC tumor region; (C and D) non-tumor thyroid tissue. Magnifications are indicated as: (A) $\mathrm{x} 100$; (B) $\mathrm{x} 200$; (C) $\mathrm{x} 100$ and (D) $\mathrm{x} 200$. PTC, papillary thyroid cancer; FOXE1, forkhead box E1.

Correlation between FOXE1 expression and the clinicopathological parameters of PTC. To examine the clinical significance of FOXE1 in PTC, samples were divided into the high and low expression groups, according to the mean value of the FOXE1 staining scores in the PTC sections (Table II). We then examined the correlation between FOXE1 and the clinicopathological parameters of PTC. Our data demonstrated that FOXE1 expression in PTC significantly correlated with the extra-capsular invasion of tumor cells, lymph node metastasis and tumor stage $(\mathrm{P}=0.048, \mathrm{P}=0.036$ and $\mathrm{P}=0.009$, respectively). No significant correlation was identified between FOXE1 expression and other clinical parameters, including age, gender, tumor size, multifocality and thyroid-stimulating hormone (TSH) level. However, we demonstrated that FOXE1 expression was significantly higher in stages III/IV compared with stages I/II of PTC $(\mathrm{P}<0.01)$. Taken together, these data suggest that FOXE1 is useful as a prognostic index for PTC.

\section{Discussion}

Transcription factors are a group of proteins that promote and regulate gene expression as well as the cell proliferation and differentiation.

A number of transcription factors belonging to the FOX family are key regulators of diverse cell functions, including oncogenesis (15), and are important in the development, invasion and metastasis of carcinoma, including FOXC1 in breast cancer (16), and FOXA1 and FOXM1 in anaplastic thyroid carcinoma $(17,18)$. However, few studies have examined the expression and function of the FOXE1 gene in PTC. In the present study, the mRNA and protein expression levels of the FOXE1 gene were investigated in Chinese patients with PTC. Out of the 26 pairs of specimens investigated by qPCR, the expression levels of FOXE1 were significantly higher in PTC 
Table II. Correlation analysis of FOXE1 expression and the clinicopathological parameters of PTC patients.

\begin{tabular}{|c|c|c|c|c|}
\hline \multirow[b]{2}{*}{ Parameter } & \multirow[b]{2}{*}{ Number (\%) } & \multicolumn{2}{|c|}{ FOXE1 expression } & \multirow[b]{2}{*}{ P-value } \\
\hline & & $\operatorname{High}(n=33)$ & Low $(n=48)$ & \\
\hline Gender & & & & 0.752 \\
\hline Male & $23(28.4)$ & 10 & 13 & \\
\hline Female & 58 (71.6) & 23 & 35 & \\
\hline Age (years) & & & & 0.346 \\
\hline$<45$ & $33(40.7)$ & 11 & 21 & \\
\hline$\geq 45$ & $48(59.3)$ & 22 & 27 & \\
\hline Tumor size (cm) & & & & 0.912 \\
\hline$\leq 2$ & $57(70.4)$ & 23 & 34 & \\
\hline$>2$ & $24(29.6)$ & 10 & 14 & \\
\hline Multifocality & & & & 0.055 \\
\hline No & $54(66.7)$ & 18 & 36 & \\
\hline Yes & $27(33.3)$ & 15 & 12 & \\
\hline Extracapsular invasion & & & & $0.048^{\mathrm{a}}$ \\
\hline No & $68(84.0)$ & 24 & 44 & \\
\hline Yes & $13(16.0)$ & 9 & 4 & \\
\hline Lymph node metastasis & & & & $0.036^{\mathrm{a}}$ \\
\hline No & $21(25.9)$ & 4 & 17 & \\
\hline Yes & $60(74.1)$ & 29 & 31 & \\
\hline Distant metastasis & & & & 1.0 \\
\hline No & $77(95.1)$ & 31 & 46 & \\
\hline Yes & $4(4.9)$ & 2 & 2 & \\
\hline Tumor stage (TNM) & & & & $0.009^{\mathrm{a}}$ \\
\hline $\mathrm{I} / \mathrm{II}$ & $55(67.9)$ & 17 & 38 & \\
\hline III/IV & $26(32.1)$ & 16 & 10 & \\
\hline TPO-Ab & & & & 0.624 \\
\hline Positive & $22(27.2)$ & 8 & 14 & \\
\hline Negative & $59(72.8)$ & 25 & 34 & \\
\hline TSH level & & & & 0.093 \\
\hline Positive & $15(18.5)$ & 9 & 6 & \\
\hline Negative & $66(81.5)$ & 24 & 42 & \\
\hline
\end{tabular}

PTC, papillary thyroid carcinoma; TSH, thyroid-stimulating hormone; TPO-Ab, thyroid peroxidase antibody; FOXE1, forkhead box E1; TSH level positive, >4.2 mIu/1; TSH level negative, 0.27-4.2 mIu/l. aSignificant difference.

tissues compared with adjacent non-tumor thyroid tissues $(\mathrm{P}<0.01)$. Similarly, data obtained from western blotting and IHC analysis were consistent with FOXE1 mRNA expression. The correlation between FOXE1 gene expression and clinical prognosis were then evaluated. Our data demonstrate that increased levels of FOXE1 expression in PTC are significantly associated with the extra-capsular invasion of tumor cells, lymph node metastasis and tumor stage, suggesting that the expression of FOXE1 is correlated with the invasion and metastasis of PTC.

Examination of the clinicopathological parameters revealed that a higher FOXE1 expression was significantly associated with advanced tumor stages and poor clinical prognosis. This hypothesis is in line with several other transcription factors of the forkhead family, including FOXA1 and FOXM1 in anaplastic thyroid carcinoma $(17,18)$. Taken together, these results suggest that the upregulation of FOXE1 may be an important event during PTC progression.

The increased expression of the thyroid-specific transcription factor, FOXE1, in PTC likely reflects the hyperactivity of the thyroid during this disease. Additionally, we demonstrated that several thyroid papillary microcarcinomas also expressed FOXE1. These observations suggest that the expression of FOXE1 occurs during the early stages of PTC development. Therefore, it is possible that the FOXE1 gene may function as an activator in PTC tumorigenesis. These results are consistent with a recently published study, which revealed that FOXE1 expression is associated with genetic 
susceptibility to PTC and forms a high-risk factor for the development of PTC (19).

In addition, there is still some debate with regard to the precise role of FOXE1 in tumorigenesis. It has been demonstrated that FOXE1 is able to function as a transcriptional repressor in cutaneous squamous cell carcinoma (9), whereas, other indirect evidence has demonstrated that FOXE1 acts as a transcriptional activator (3). Our data indicated that FOXE1 expression is elevated in PTC samples, suggesting that FOXE1 gene expression may be associated with a high risk of developing PTC.

Notably, our results have demonstrated that FOXE1 expression is upregulated at the mRNA and protein levels in PTC tissues. Although the sample size in this study was small and the findings cannot be generalized to the broader population, we hypothesize that FOXE1 may function as a positive regulator in thyroid carcinogenesis. However, it remains unclear whether the activation of certain pathways, including the Sonic Hedgehog (SHh) pathway, in thyroid cancer contribute to FOXE1 transcriptional activity and subsequently lead to an increased expression of FOXE1 (20).

By contrast, the TSH signaling cascade, which is present in thyroid follicular cells is important in the cell differentiation of the thyroid gland. The pathway is initiated by TSH binding to its transmembrane receptor, leading to the release of adenosine cyclophosphate (cAMP) and protein kinase A (PKA), which subsequently activate thyroid-specific transcription factors, including PAX-8, TTF1 and FOXE1. In the present study, based on the preoperatively evaluated expression levels of TSH, we retrospectively investigated whether TSH expression correlated with FOXE1, and thus may result in higher expression levels of FOXE1 in certain PTC cases. However, although a positive correlation was observed, the correlation between TSH levels and FOXE1 expression was not statistically significant. The precise role and molecular mechanism responsible for the potential tumor activator role of FOXE1 in PTC requires additional investigation, and may have potential clinical implications for the clinical prognosis and therapy of PTC.

To the best of our knowledge, this is the first study to report the mRNA and protein expression levels of FOXE1 in PTC and analyze its clinical significance. In conclusion, we have demonstrated that the FOXE1 gene exhibits significant differential expression levels between PTC tissues and adjacent non-tumor thyroid tissues. Our results suggest that FOXE1 may be important in the development of PTC and may be a candidate prognostic biomarker and a new therapeutic target.

\section{References}

1. Davies L and Welch HG: Increasing incidence of thyroid cancer in the United States, 1973-2002. JAMA 295: 2164-2167, 2006.
2. Hay ID, Thompson GB, Grant CS, et al: Papillary thyroid carcinoma managed at the Mayo Clinic during six decades (1940-1999): temporal trends in initial therapy and long-term outcome in 2444 consecutively treated patients. World J Surg 26: 879-885, 2002.

3. Zannini M, Avantaggiato V, Biffali E, et al: TTF-2, a new forkhead protein, shows a temporal expression in the developing thyroid which is consistent with a role in controlling the onset of differentiation. EMBO J 16: 3185-3197, 1997.

4. Katoh M and Katoh M: Human FOX gene family (Review). Int J Oncol 25: 1495-1500, 2004.

5. Dathan N, Parlato R, Rosica A, De Felice M and Di Lauro R: Distribution of the titf2/foxel gene product is consistent with an important role in the development of foregut endoderm, palate and hair. Dev Dyn 224: 450-456, 2002.

6. De Felice M, Ovitt C, Biffali E, et al: A mouse model for hereditary thyroid dysgenesis and cleft palate. Nat Genet 19: 395-398, 1998.

7. Brune K, Hong SM, Li A, et al: Genetic and epigenetic alterations of familial pancreatic cancers. Cancer Epidemiol Biomarkers Prev 17: 3536-3542, 2008

8. Weisenberger DJ, Trinh BN, Campan M, et al: DNA methylation analysis by digital bisulfite genomic sequencing and digital MethyLight. Nucleic Acids Res 36: 4689-4698, 2008.

9. Venza I, Visalli M, Tripodo B, Lentini M, Teti D and Venza M: Investigation into FOXE1 genetic variations in cutaneous squamous cell carcinoma. Br J Dermatol 162: 681-683, 2010.

10. Sequeira MJ, Morgan JM, Fuhrer D, Wheeler MH, Jasani B and Ludgate M: Thyroid transcription factor-2 gene expression in benign and malignant thyroid lesions. Thyroid 11: 995-1001, 2001.

11. Gudmundsson J, Sulem P, Gudbjartsson DF, et al: Common variants on $9 \mathrm{q} 22.33$ and $14 \mathrm{q} 13.3$ predispose to thyroid cancer in European populations. Nat Genet 41: 460-464, 2009.

12. Nam KH, Noh TW, Chung SH, et al: Expression of the membrane mucins MUC4 and MUC15, potential markers of malignancy and prognosis, in papillary thyroid carcinorma. Thyroid 21: 745-750, 2011.

13. Shaha AR: TNM classification of thyroid carcinoma. World J Surg 31: 879-887, 2007.

14. Cooper DS, Doherty GM, Haugen BR, et al; American Thyroid Association (ATA) Guidelines Taskforce on Thyroid Nodules and Differentiated Thyroid Cancer: Revised American Thyroid Association management guidelines for patients with thyroid nodules and differentiated thyroid cancer. Thyroid 19: 1167-1214, 2009.

15. Hannenhalli S and Kaestner KH: The evolution of Fox genes and their role in development and disease. Nat Rev Genet 10: 233-240, 2009.

16. Sizemore ST and Keri RA: The forkhead box transcription factor FOXC1 promotes breast cancer invasion by inducing matrix metalloprotease 7 (MMP7) expression. J Biol Chem 287: 24631-24640, 2012.

17. Nucera C, Eeckhoute J, Finn S, et al: FOXA1 is a potential oncogene in anaplastic thyroid carcinoma. Clin Cancer Res 15: 3680-3689, 2009.

18. Bellelli R, Castellone MD, Garcia-Rostan G, et al: FOXM1 is a molecular determinant of the mitogenic and invasive phenotype of anaplastic thyroid carcinoma. Endocr Relat Cancer 19: 695-710, 2012.

19. Matsuse M, Takahashi M, Mitsutake N, et al: The FOXE1 and NKX2-1 loci are associated with susceptibility to papillary thyroid carcinoma in the Japanese population. J Med Genet 48: 645-648, 2011

20. Xu X, Ding H, Rao G, et al: Activation of the Sonic Hedgehog pathway in thyroid neoplasms and its potential role in tumor cell proliferation. Endocr Relat Cancer 19: 167-179, 2012. 